\title{
Transepithelial potential difference in cystic fibrosis
}

\author{
JG HAY, DM GEDDES
}

From Brompton Hospital, London

\begin{abstract}
In cystic fibrosis the potential difference between interstitial fluid and the surface of respiratory and sweat duct epithelia is abnormally large. This study attempted to confirm this finding and investigate its value for a diagnostic test. With a subcutaneous reference electrode the mean potential difference of the inferior turbinate was $-25 \mathrm{mv}$ in 19 patients with cystic fibrosis, which was significantly greater than the difference of $-13 \mathrm{mv}$ in 22 normal subjects $(\mathrm{p}<0.02)$. Of the observations in the patients with cystic fibrosis, $67 \%$ were greater than the largest potential difference observed in normal individuals. Potential differences from the lip and forearm were also significantly greater in cystic fibrosis than in normal subjects, but there was considerable overlap. Changes in the electrical properties of nasal epithelium and skin have been confirmed in cystic fibrosis, but these have limited use for diagnosis.
\end{abstract}

Abnormalities of the electrical properties of two epithelial surfaces have recently been described in patients with cystic fibrosis. The first description was of an increased negative potential across nasal respiratory epithelium.' Subsequently, increased negative potentials across sweat duct epithelium have been described ${ }^{23}$ both in isolated sweat glands and in vivo.

The work on sweat ducts has strown the basic abnormality of function to be an impermeability of the epithelium to chloride ions. This explains both the electrolyte and the electrical disturbance of sweat ducts in cystic fibrosis. The same defect is probably the major explanation of the abnormal electrical properties of the respiratory epithelia, although abnormal sodium transport may also contribute. $^{4}$

The observations may have diagnostic importance, and further investigation may lead to a clearer understanding of the pathogenesis of cystic fibrosis. Airway humidity and osmolarity may play an important part in many aspects of airway function, ${ }^{5}$ in particular controlling the composition of airway fluid, which may itself influence mucociliary clearance.

We have measured the potential difference between nasal epithelia and interstitial fluid in a group of normal subjects and patients with cystic fibrosis.

Address for reprint requests: Dr JG Hay, Brompton Hospital, London SW3 6HP.

Accepted 11 February 1985
We have also observed the potential differences generated at several skin sites.

\section{Methods}

The equipment consisted of a high impedance voltmeter $\left(10^{11}\right.$ ohms) connected to a Devices recorder. The voltmeter was connected by the means of calomel cells and saturated potassium chloride bridges to a reference and recording electrode. The reference electrode was a gauge 20 Abbocath cannula placed subcutaneously in the left forearm and slowly perfused with Ringers saline (Travenol) from a Soluset IV chamber. The recording electrode was a gauge 16 Abbocath cannula filled with Ringers saline in $1 \%$ agar and also slowly perfused with Ringers saline in a similar manner.

Measurements were made at the tip of the inferior turbinate and at a distance $1-2 \mathrm{~cm}$ distal to the tip along the inferior surface of the turbinate. The tip of the inferior turbinate is an area covered with squamous epithelium, and the inferior surface is covered in ciliated respiratory epithelium.' These sites were visualised with the aid of an oroscope speculum and a bright light. Measurements were also made at the following skin sites after cleansing them with alcohol: right anterior forearm, cheek, external nares, and lip. The lip is an area of skin devoid of sweat ducts.

From two to six stable potentials of at least 15 seconds' duration were recorded from each site in every individual. The mean value from each site was 
then calculated. The electrodes were tested for asymmetry before and after each subject and values of greater than $3 \mathrm{mv}$ were not seen.

Observations were made in 22 normal subjects and in 19 patients fulfilling the diagnosis of cystic fibrosis on clinical and sweat test criteria. The mean age for normal subjects was 29 (SD 4.8) years; eight were men and 14 women. Four of the women were taking oral contraceptives and another atenolol. The cystic fibrosis group had a mean age of 21 (3.8) years and comprised 16 men and three women. All of those with cystic fibrosis were taking pancreatic supplements and antibiotic treatment; all but four were having regular treatment with an inhaled $\beta$ agonist.

All subjects gave their informed consent. The data were analysed for significant difference by means of the Mann-Whitney $U$ test.

\section{Results}

Potential differences from all the sites studied are summaried in the table. Figure 1 shows the mean values obtained from each individual at the arm, lip, and tip of the inferior turbinate. The mean value for the group is also shown with one standard error above and below the mean. Values from the arm and lip are significantly more negative in patients with cystic fibrosis than in normal individuals, but the overlap is considerable.

From the results of the distal site of the turbinate (fig 2), it can be seen that the normal population has a range from -8 to -20 with a mean value of $-13 \mathrm{mv}$. Patients with cystic fibrosis have a large scatter but $67 \%$ have values above the highest normal value, the mean value being $-25 \mathrm{mv}$. If the observations of the distal site on the turbinate are analysed in a different manner by taking only the most negative stable potential that was recorded from each individual at this site rather than the mean of the observations recorded (fig 2), the mean value becomes $-17 \mathrm{mv}$ for the normal group and

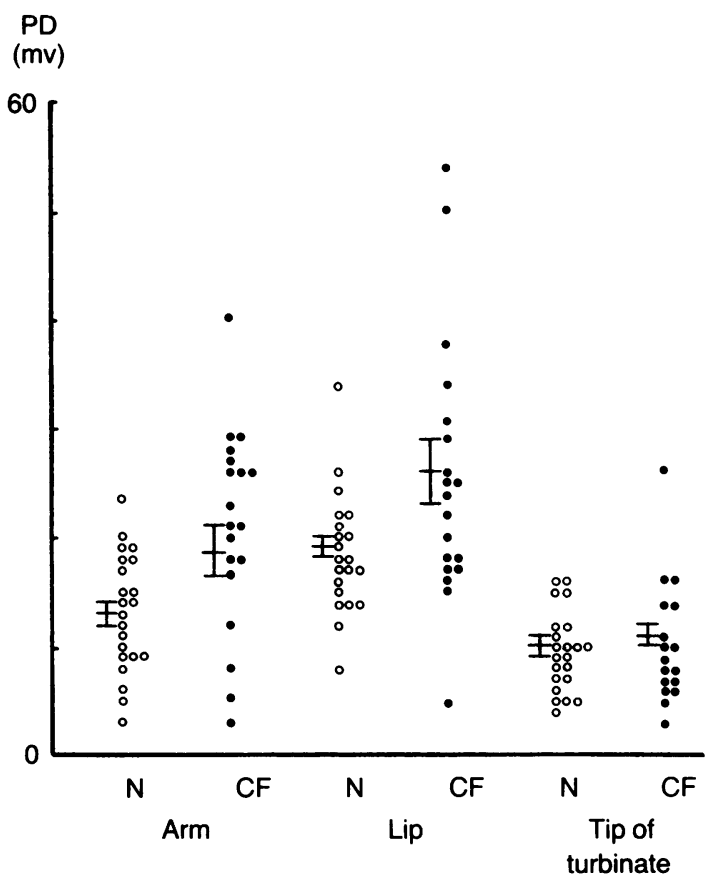

Fig 1 Potential differences between the interstitial fuid and the surface of the arm, lip, and tip of the inferior turbinate. Each point represents the mean of two to six measurements at each site in an individual (open circles normal subjects $(N)$, closed circles patients with cystic fibrosis (CF). The horizontal bars represent mean values and one standard error.

$-36 \mathrm{mv}$ for those with cystic fibrosis. These values correspond reasonably with the observations of Knowles et al ${ }^{1}$ of -24 and -53 mv respectively.

No clear relationship could be seen beween potential difference and an individual's age, sex, or drug treatment; the age distribution of both groups was narrow, however, and the number of patients with cystic fibrosis not taking $\beta$ agonists was small.

Potential difference between the different sites and the interstitial fuid in normal individuals and patients with cystic fibrosis (CF)

\begin{tabular}{|c|c|c|c|}
\hline \multirow[t]{2}{*}{ Site } & \multicolumn{3}{|c|}{ Mean (SD) transepithelial potential difference ( $m v)$} \\
\hline & Normal $(n=22)$ & $C F(n=19)$ & $p^{*}$ \\
\hline $\begin{array}{l}\text { Arm } \\
\text { Cheek } \\
\text { Lip } \\
\text { Nose } \\
\text { Tip of inferior turbinate } \\
\text { Distal inferior turbinate } \\
\text { Most negative value on inferior turbinate }\end{array}$ & $\begin{array}{l}13(5.4) \\
14(4.5) \\
19(5.4) \\
19(6.3) \\
10(3.7) \\
13(4.6) \\
17(5.1)\end{array}$ & $\begin{array}{l}21(9.3) \\
14(6.0) \\
26(11.8) \\
17(6.4) \\
10(5.5) \\
25(13.3) \\
36(18.6)\end{array}$ & $\begin{array}{l}<0.01 \\
\text { NS } \\
<0.05 \\
\text { NS } \\
\text { NS } \\
<0.02 \\
<0.02\end{array}$ \\
\hline
\end{tabular}

* Mann-Whitney test.

NS-not significant. 


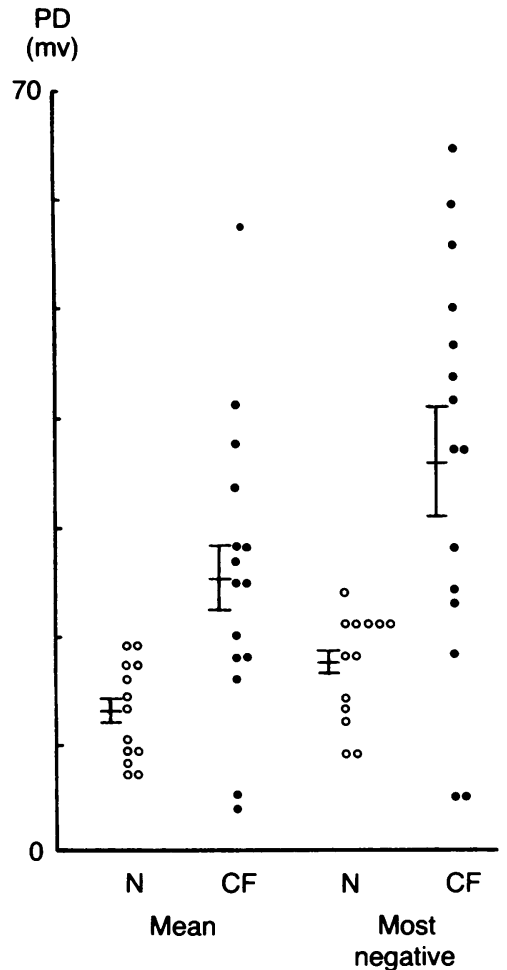

Fig 2 Potential differences measured along the inferior surface of the inferior turbinate for normal individuals ( $N$-open circles) and patients with cystic fibrosis (CF-closed circles). The data have been analysed in two ways: on the left hand diagram as in the previous figure, each point representing the mean of several observations in an individual, and in the right hand diagram with only the most negative stable potential from each individual shown.

\section{Discussion}

We have confirmed the finding of an increased negative potential generated by the inferior surface of the inferior turbinate in patients with cystic fibrosis. Our findings are different from those of Knowles' in two respects. Firstly, we have observed some overlap of the cystic fibrosis and normal ranges. Secondly, we have noted smaller potential differences for both the normal and the cystic fibrosis groups. Possibly some of the cystic fibrosis measurements fell within the normal range because some recordings were made from regions that had been denuded of epithelium or had suffered squamous metaplasia, changes that would not be visually evident. Nasal disease is common in cystic fibrosis, and only areas of ciliated respiratory epithelium show the electrical abnormality. Potentials from the tip of the turbinate, which is covered in squamous epithelia, are similar in the two groups and small in magnitude. Furthermore, Knowles has shown that abrasion of the mucosa eliminates the potential difference.'

When we analysed our results from the distal site on the turbinate (fig 2 ) by taking only the most negative stable potentials from each patient rather than their means, the group means rose considerably-30\% and $48 \%$ respectively for normal subjects and patients with cystic fibrosis-to values similar to those of Knowles, without disturbing the pattern of $67 \%$ of cystic fibrosis observations being above the highest normal value. Possibly in some patients distal measurements were not obtained at all as a result of difficulties in placing the recording electrode, and in others non-distal measurements reduced the mean values. This measurement is not sufficiently sensitive to replace the sweat test, but we have found a clear upper limit of normal nasal potentials exceeded by $67 \%$ of patients with cystic fibrosis. This may be of some clinical value, especially since our patients were of an age where sweat test results, before attempts at fludrocortisone suppression are made, are often ambiguous.

We also looked at several skin sites to see whether potentials from sites which were technically easier to measure showed the same abnormality. Quinton showed that sweat ducts of patients with cystic fibrosis have higher negative potentials. ${ }^{23}$ Schulz suggested that higher skin potentials may be found in cystic fibrosis after sweating is induced, ${ }^{6}$ and Edmunds also recalled noting an increased perianal skin potential in cystic fibrosis.?

We have found skin potentials to have a large scatter with considerable overlapping of the cystic fibrosis and normal groups. Apart from measurements at the external nares, however, skin potentials from patients with cystic fibrosis were more negative than those from normal subjects. The difference reached significance in the case of the arm and the lip. The considerable overlapping of normal and cystic fibrosis values, however, makes skin potential measurements of little value as a diagnostic test. The wide scatter of observations is not surprising given the complexity of the electrical properties of the skin, which have been extensively reviewed in psychophysiological publications. ${ }^{8}$ In brief, the skin may be regarded as consisting of two batteries, the epidermal cells and the sweat ducts, operating in parallel, each with its own variable resistance dependent on the extent of filling of the sweat duct with sweat and the hydration of the corneum. In our studies neither the rate of sweating nor the hydration of the corneum was taken into account.

The observations on the lip are of particular interest as the lip is devoid of sweat ducts and the potential observed may be assumed to be derived from the 
epidermal cells. The finding of a significantly more negative potential at this site in individuals with cystic fibrosis suggests that epidermal cells may also possess the electrical abnormality seen in the respiratory tract and sweat duct epithelia. Abnormal electrical potentials are therefore produced by respiratory, sweat duct, and lip epithelia in cystic fibrosis. Furthermore, renal handling of sodium may be abnormal." Although these findings may have little diagnostic value, they do suggest a widespread epithelial ion transport defect that may be fundamental to the pathogenesis of the disorder.

We would like to thank Dr JC Batten for allowing us to study some of his patients, Mr Logan Sinclair for technical assistance, and Miss A Betchley for typing the manuscript.

\section{References}

1 Knowles M, Gatzy J, Boucher R. Increased bio-electric potential difference across respiratory epithelia in cystic fibrosis. N Engl J Med 1981;305: 1489-95.

2 Quinton PM. Chloride impermeability in cystic fibrosis. Nature 1983;301:421-2.

3 Quinton PM, Bijman J. Higher bio-electric potentials due to decreased chloride absorption in the sweat glands of patients with cystic fibrosis. $N$ Engl J Med 1983;308: 1185-9.

4 Knowles M, Gatzy J, Boucher R. Relative ion permeability of normal and cystic fibrosis nasal epithelium. $J$ Clin Invest 1983;71:1410-7.

5 Hogg JC. Is asthma an epithelial disease? Am Rev Respir Dis 1984;129:207-8.

6 Schulz IJ, Fromter E. Mikropunktionsuntersuchungen an Schweissdrusen von Mucoviscidosepatienten und gesunden Versuchspersonen. In: Mucoviscidose, Cystiche Fibrose: 2 Deutsches Symposiom. Stuttgart: Georg Thieme, 1968:12-21.

7 Edmunds CJ. Skin electrical potential difference in cystic fibrosis. Lancet 1983; ;:937-8.

8 Edelberg R. Biopotentials from the skin surface: the hydration effect. Ann NY Acad Sci 1968;148:252-62.

9 Kusoffsky UBE, Strandvik B. Renal function in cystic fibrosis with special reference to the renal sodium handling. Acta Paediatr Scand 1982;71:833-8. 